\title{
COLLEGE NEWS
}

(J Bangladesh Coll Phys Surg 2016; 34: 233-242)

College news Examinations news: Results of FCPS Part-I, Part-II and MCPS examination held in July are given bellow:

3248 candidates appeared in FCPS Part-I, examinatin held in July, 2016 of which 272 candidates came out successful. Subject wise results are as follows:

Result of FCPS Part-I Examination (July, 2016)

\begin{tabular}{|c|c|c|c|c|}
\hline \multirow[t]{2}{*}{ SL. No. } & \multirow[t]{2}{*}{ Subject } & \multicolumn{3}{|c|}{ July-2015 } \\
\hline & & Total Candidate & Total Passed & $\begin{array}{c}\text { Percentage } \\
\%\end{array}$ \\
\hline$\overline{1 .}$ & Anaesthesiology & 109 & 8 & 7.34 \\
\hline 2. & Biochemistry & 5 & 0 & 0.00 \\
\hline 3. & Dentistry & 193 & 3 & 1.55 \\
\hline 4. & Dermatology \& Venereology & 47 & 3 & 6.38 \\
\hline 5. & Family Medicine & 2 & 0 & 0.00 \\
\hline 6. & Haematology & 13 & 2 & 15.38 \\
\hline 7. & Histopathology & 13 & 0 & 0.00 \\
\hline 8. & Medicine & 920 & 59 & 6.41 \\
\hline 9. & Microbiology & 16 & 1 & 6.25 \\
\hline 10. & Obst. \& Gynae & 704 & 105 & 14.91 \\
\hline 11. & Ophthalmology & 84 & 21 & 25.00 \\
\hline 12. & Otolaryngology & 89 & 4 & 4.49 \\
\hline 13. & Paediatrics & 359 & 41 & 11.42 \\
\hline 14. & Physical Medicine \& Rehabilitation & 30 & 1 & 3.33 \\
\hline 15. & Psychiatry & 6 & 2 & 33.33 \\
\hline 16. & Radiology \& Imaging & 34 & 4 & 11.76 \\
\hline 17. & Radiotherapy & 35 & 7 & 20.00 \\
\hline 18. & Surgery & 589 & 11 & 1.87 \\
\hline \multirow[t]{2}{*}{19} & Transfusion Medicine & & & \\
\hline & Total & 3248 & 272 & 8.37 \\
\hline
\end{tabular}

The following candidates satisfied the Board of Examiners and are declared to have passed the FCPS - II Examinations held in July, 2016 subject to confirmation by the council of Bangladesh College of Physicians and Surgeons

\begin{tabular}{llll}
\hline Roll No. & Name & From where graduated & Subject \\
\hline Roll No & Name & From Where Graduate & Subject \\
130001 & Umme Habiba Ferdaushi & Sher-E-Bangla Medical College, Barisal & Cardiology \\
140001 & Md. Abdul Hannan & MAG Osmani Medical College, Sylhet & Cardiovascular Surgery \\
140002 & Md Faizus Sazzad & Dinajpur Medical College, Dinajpur & Cardiovascular Surgery \\
170001 & Hurjahan Banu & Khulna Medical College, Khulna & Endocrinology and Metabolism \\
550001 & Dr. Mst. Tajmira Sultana & Sir Salimullah Medical College, Dhaka & Feoto-Maternal Medicine \\
\hline
\end{tabular}




\begin{tabular}{|c|c|c|c|}
\hline Roll No. & Name & From where graduated & Subject \\
\hline 290001 & Md. Shariful Islam & Shaheed Ziaur Rahman Medical College, Bogra & Neuro-Surgery \\
\hline 290002 & Patoary Mohammed Faruque & Sir Salimullah Medical College, Dhaka & Neuro-Surgery \\
\hline 340001 & Abul Kalam Azad & Mymensingh Medical College, Mymensingh & Orthopaedic Surgery \\
\hline 340002 & Mirza Osman Beg & Comilla Medical College, Comilla & Orthopaedic Surgery \\
\hline 540001 & Bithi Debnath & Dhaka Medical College, Dhaka & Paediatric Neurology \& Development \\
\hline 410001 & Iqbal Ahmed & Rajshahi Medical College, Rajshahi & Plastic and Reconstructive Surgery \\
\hline 410003 & Md. Shahin Shah & Rangpur Medical College, Rangpur & Plastic and Reconstructive Surgery \\
\hline 410004 & Mst. Masuma Sarker & Dhaka Medical College, Dhaka & Plastic and Reconstructive Surgery \\
\hline 410005 & Afrina Sharmin & Z.H. Sikder Women $\measuredangle$ s Medical College, Dhaka & Plastic and Reconstructive Surgery \\
\hline 500002 & Dr. Ujjal Barua & Comilla Medical College, Comilla & Urology \\
\hline 500008 & Shafiqur Rahman & Sir Salimullah Medical College, Dhaka & Urology \\
\hline 110004 & Debashish Das & Jahurul Islam Medical College, Bajitpur & Anaesthesiology \\
\hline 110010 & Khalid Nur Md. Mahbub & Sir Salimullah Medical College, Dhaka & Anaesthesiology \\
\hline 110013 & Raju Ahmed & Sher-E-Bangla Medical College, Barisal & Anaesthesiology \\
\hline 110015 & Md. Ahsanul Kabir & Sir Salimullah Medical College, Dhaka & Anaesthesiology \\
\hline 110018 & Md. Abdul Jabbar & Mymensingh Medical College, Mymensingh & Anaesthesiology \\
\hline 150001 & Dr.Sultana Parveen & Dhaka Dental College, Dhaka & Conservative Dentistry and Endodontics \\
\hline 150002 & Shiren Sultana & Pioneer Dental College, Dhaka & Conservative Dentistry and Endodontics \\
\hline 160001 & Bilqis Akter & Sher-E-Bangla Medical College, Barisal & Dermatology and Venereology \\
\hline 240013 & Israt Zerin Eva & Dhaka Medical College, Dhaka & Medicine \\
\hline 240016 & Shuvojit Sen & Armed Forces Medical College, Dhaka & Medicine \\
\hline 240031 & Tania Tajreen & Chittagong Medical College, Chittagong & Medicine \\
\hline 240044 & Rozina Sultana & Rangpur Medical College, Rangpur & Medicine \\
\hline 240056 & Dr.Atiquzzaman & Dhaka National Medical College, Dhaka & Medicine \\
\hline 240070 & Md. Ajmirul Hoque Sarkar & Rajshahi Medical College, Rajshahi & Medicine \\
\hline 240071 & Nasir Uddin Ahmed & Chittagong Medical College, Chittagong & Medicine \\
\hline 240086 & Ishrat Binte Reza & Bangladesh Medical College, Dhaka & Medicine \\
\hline 240095 & $\begin{array}{l}\text { Mohammad Kafil Uddin } \\
\text { Chowdhery }\end{array}$ & Chittagong Medical College, Chittagong & Medicine \\
\hline 240104 & Abhizit Pandit & Sir Salimullah Medical College, Dhaka & Medicine \\
\hline 240107 & Dr. Mostofa Kamal Chowdhury & Dhaka Medical College, Dhaka & Medicine \\
\hline 240112 & Dr. Suman Kanti Chowdhury & Dhaka Medical College, Dhaka & Medicine \\
\hline 240121 & Mazharul Islam & Sher-E-Bangla Medical College, Barisal & Medicine \\
\hline 240123 & Shabnam Jahan Hoque & Dhaka Medical College, Dhaka & Medicine \\
\hline 240125 & Md. Mahbubul Alam & Mymensingh Medical College, Mymensingh & Medicine \\
\hline 240126 & Hasan Hafizur Rahman & Dhaka Medical College, Dhaka & Medicine \\
\hline 240131 & Dr. Mrinmay Kumar Podder & Mymensingh Medical College, Mymensingh & Medicine \\
\hline 240139 & Humayra Jesmin & Rajshahi Medical College, Rajshahi & Medicine \\
\hline 240140 & $\begin{array}{l}\text { Muhammad Abdullah } \\
\text { Jahed Khan }\end{array}$ & Chittagong Medical College, Chittagong & Medicine \\
\hline 240150 & Md. Moniruzzaman Asraf & Rajshahi Medical College, Rajshahi & Medicine \\
\hline 240167 & Md. Saiful Malek & Rajshahi Medical College, Rajshahi & Medicine \\
\hline
\end{tabular}




\begin{tabular}{|c|c|c|c|}
\hline Roll No. & Name & From where graduated & Subject \\
\hline 240177 & Soumitra Roy & MAG Osmani Medical College, Sylhet & Medicine \\
\hline 240182 & Md. Shyfullah & Rajshahi Medical College, Rajshahi & Medicine \\
\hline 240210 & Md. Amzad Hossain & Mymensingh Medical College, Mymensingh & Medicine \\
\hline 240212 & Tridip Kanti Barman & Sir Salimullah Medical College, Dhaka & Medicine \\
\hline 240213 & Ashim Kumar Saha & Khulna Medical College, Khulna & Medicine \\
\hline 240215 & Md. Naheed Hasan & Dhaka Medical College, Dhaka & Medicine \\
\hline 240218 & Fouzia Anar & Dhaka Medical College, Dhaka & Medicine \\
\hline 240221 & Shantonu Kumer Saha & Dhaka Medical College, Dhaka & Medicine \\
\hline 240222 & Dr. Goutam Talukder & MAG Osmani Medical College, Sylhet & Medicine \\
\hline 240234 & Sanjoy Kumer Paul & Chittagong Medical College, Chittagong & Medicine \\
\hline 240235 & Nira Ferdous & Z.H. Sikder Women ¿s Medical College, Dhaka & Medicine \\
\hline 240251 & Azimun Nessa & Mymensingh Medical College, Mymensingh & Medicine \\
\hline 240252 & Rubyat Hasan Chowdhury & Mymensingh Medical College, Mymensingh & Medicine \\
\hline 240262 & Mohammad Kamrul Islam & MAG Osmani Medical College, Sylhet & Medicine \\
\hline 240265 & Mohammad Saydur Rahman & Mymensingh Medical College, Mymensingh & Medicine \\
\hline 240280 & Dr. Md. Mahidul Alam & Rajshahi Medical College, Rajshahi & Medicine \\
\hline 240294 & Md. Ruhul Amin Sarkar & Sir Salimullah Medical College, Dhaka & Medicine \\
\hline 240307 & Meherunnesa Mukta & Rangpur Medical College, Rangpur & Medicine \\
\hline 240314 & $\begin{array}{l}\text { Md. Mamun-Or-Rashid } \\
\text { Ibne Harun }\end{array}$ & Mymensingh Medical College, Mymensingh & Medicine \\
\hline 240315 & $\begin{array}{l}\text { Abu Hayat Mohammod } \\
\text { Waliur Rahman }\end{array}$ & Sir Salimullah Medical College, Dhaka & Medicine \\
\hline 240318 & Faria Quadir & Sher-E-Bangla Medical College, Barisal & Medicine \\
\hline 240321 & Afsana Rahman & Dhaka Medical College, Dhaka & Medicine \\
\hline 240323 & Dr. Milton Barua & Chittagong Medical College, Chittagong & Medicine \\
\hline 240330 & Mohammad Jane Alam & Sher-E-Bangla Medical College, Barisal & Medicine \\
\hline 240340 & Md. Jahangir Kabir & Khulna Medical College, Khulna & Medicine \\
\hline 240341 & Dr. J.M. Ariful Islam & Mymensingh Medical College, Mymensingh & Medicine \\
\hline 240355 & Md. Ashiq Iqbal & Rajshahi Medical College, Rajshahi & Medicine \\
\hline 240357 & Mohammad Shohel Khan & Shaheed Ziaur Rahman Medical College, Bogra & Medicine \\
\hline 240358 & Muhammed Saiful Islam & Sir Salimullah Medical College, Dhaka & Medicine \\
\hline 240365 & Muhammad Ataul Gani Osmani & Sir Salimullah Medical College, Dhaka & Medicine \\
\hline 240366 & Issa Muhammad Baker & Shaheed Ziaur Rahman Medical College, Bogra & Medicine \\
\hline 240367 & Md. Habibul Ghani & Mymensingh Medical College, Mymensingh & Medicine \\
\hline 240368 & Dr. Anupam Das & Rangpur Medical College, Rangpur & Medicine \\
\hline 240369 & Rahatun Nayeem & Sir Salimullah Medical College, Dhaka & Medicine \\
\hline 240375 & Z.H.M.Nazmul Alam & MAG Osmani Medical College, Sylhet & Medicine \\
\hline 240377 & Abdullah Al-Muti & Rangpur Medical College, Rangpur & Medicine \\
\hline 240400 & Dr. B.U.M Wahid Ahmed & MAG Osmani Medical College, Sylhet & Medicine \\
\hline 240429 & Md Elias Bhuiyan & Sir Salimullah Medical College, Dhaka & Medicine \\
\hline 240439 & Mohammad Lokman Hakim & Chittagong Medical College, Chittagong & Medicine \\
\hline 240455 & Rozana Rouf & MAG Osmani Medical College, Sylhet & Medicine \\
\hline 240462 & Amrita Kumar Deb Nath & Dhaka Medical College, Dhaka & Medicine \\
\hline
\end{tabular}




\begin{tabular}{|c|c|c|c|}
\hline Roll No. & Name & From where graduated & Subject \\
\hline 240498 & Pritish Tarafder & Khulna Medical College, Khulna & Medicine \\
\hline 240502 & Md. Abdul Hamid & Chittagong Medical College, Chittagong & Medicine \\
\hline 250001 & Dr. Rezina Jasmine & Rajshahi Medical College, Rajshahi & Microbiology \\
\hline 300033 & Bilkish Jahan Ferdushy & Armed Forces Medical College, Dhaka & Obst and Gynae \\
\hline 300069 & Salma Akter & Sir Salimullah Medical College, Dhaka & Obst and Gynae \\
\hline 300072 & Noor-E-Naharin & Rajshahi Medical College, Rajshahi & Obst and Gynae \\
\hline 300077 & Nazmun Nahar & Sir Salimullah Medical College, Dhaka & Obst and Gynae \\
\hline 300087 & Umme Rehnuma Tarannum & Chittagong Medical College, Chittagong & Obst and Gynae \\
\hline 300089 & Fahmida Hoque & Chittagong Medical College, Chittagong & Obst and Gynae \\
\hline 300098 & Sabiha Shimul & Dinajpur Medical College, Dinajpur & Obst and Gynae \\
\hline 300115 & Mridula Kar & Sher-E-Bangla Medical College, Barisal & Obst and Gynae \\
\hline 300116 & Farhana Parveen & Sher-E-Bangla Medical College, Barisal & Obst and Gynae \\
\hline 300132 & Dr. Umme Abedin Saima & Sher-E-Bangla Medical College, Barisal & Obst and Gynae \\
\hline 300138 & Kaoser Jahan & Mymensingh Medical College, Mymensingh & Obst and Gynae \\
\hline 300140 & Ummay Salma & Sher-E-Bangla Medical College, Barisal & Obst and Gynae \\
\hline 300145 & Ainun Nahar & Sir Salimullah Medical College, Dhaka & Obst and Gynae \\
\hline 300158 & Ruma Afrose & Rangpur Medical College, Rangpur & Obst and Gynae \\
\hline 300176 & Dr. Mousumi Saha & MAG Osmani Medical College, Sylhet & Obst and Gynae \\
\hline 300187 & Laila Kamruzzahan Panna & Rangpur Medical College, Rangpur & Obst and Gynae \\
\hline 300191 & Saida Bilkis Khanam & Sir Salimullah Medical College, Dhaka & Obst and Gynae \\
\hline 300203 & Ivy Nasrin & Dhaka Medical College, Dhaka & Obst and Gynae \\
\hline 300207 & Golshan Ara Kohinoor & MAG Osmani Medical College, Sylhet & Obst and Gynae \\
\hline 300210 & Abanti Ghosh & Rajshahi Medical College, Rajshahi & Obst and Gynae \\
\hline 300213 & Mitheel-Ibna Islam & Rangpur Medical College, Rangpur & Obst and Gynae \\
\hline 300221 & Shamima Amir & Mymensingh Medical College, Mymensingh & Obst and Gynae \\
\hline 300223 & Mst. Shabrin Akhter & Sir Salimullah Medical College, Dhaka & Obst and Gynae \\
\hline 300239 & Sabah Sultana & Rajshahi Medical College, Rajshahi & Obst and Gynae \\
\hline 300247 & Iffana Azam & Jalalabad Ragib-Rabeya Medical College, Sylhet & Obst and Gynae \\
\hline 300253 & Manjan Ara & Rajshahi Medical College, Rajshahi & Obst and Gynae \\
\hline 300258 & Lutfunnahar Shampa & Dhaka Medical College, Dhaka & Obst and Gynae \\
\hline 300265 & Dina Layla Hossain & Dhaka Medical College, Dhaka & Obst and Gynae \\
\hline 300268 & Ashfi Laila Elora & Mymensingh Medical College, Mymensingh & Obst and Gynae \\
\hline 300277 & Farhana Haque Choudhury & Rangpur Medical College, Rangpur & Obst and Gynae \\
\hline 300283 & Ummey Nazmin Islam & Dhaka Medical College, Dhaka & Obst and Gynae \\
\hline 300286 & Syeda Farhana Ali & Bangladesh Medical College, Dhaka & Obst and Gynae \\
\hline 300288 & Farhana Ahmed Nancy & Bangladesh Medical College, Dhaka & Obst and Gynae \\
\hline 300300 & Farzana Nasrin & Sir Salimullah Medical College, Dhaka & Obst and Gynae \\
\hline 300301 & Farzana Islam Khan & MAG Osmani Medical College, Sylhet & Obst and Gynae \\
\hline 300312 & Nafisa Jesmin & MAG Osmani Medical College, Sylhet & Obst and Gynae \\
\hline 300314 & Panchami Goshwami & Dinajpur Medical College, Dinajpur & Obst and Gynae \\
\hline 300322 & Lucky Rahman & Sir Salimullah Medical College, Dhaka & Obst and Gynae \\
\hline 300327 & Fatema Islam & Sir Salimullah Medical College, Dhaka & Obst and Gynae \\
\hline
\end{tabular}




\begin{tabular}{|c|c|c|c|}
\hline Roll No. & Name & From where graduated & Subject \\
\hline 300336 & Fatema Begum & Faridpur Medical College, Faridpur & Obst and Gynae \\
\hline 300341 & Tania Akbar & International Medical College, Tongi, Dhaka & Obst and Gynae \\
\hline 300343 & Sanjida Khan & Jahurul Islam Medical College, Bajitpur & Obst and Gynae \\
\hline 300350 & Dr. Sharmin Akhter Shumi & Sher-E-Bangla Medical College, Barisal & Obst and Gynae \\
\hline 300351 & Mst. Taslima Akther & Chittagong Medical College, Chittagong & Obst and Gynae \\
\hline 300363 & Anika Tabassum & Dhaka Medical College, Dhaka & Obst and Gynae \\
\hline 300371 & Shahida Akter & Sir Salimullah Medical College, Dhaka & Obst and Gynae \\
\hline 300374 & Salma Akter & Dhaka Medical College, Dhaka & Obst and Gynae \\
\hline 300378 & Rumana Akhter & Shaheed Ziaur Rahman Medical College, Bogra & Obst and Gynae \\
\hline 300385 & Naz Yasmin & Dhaka Medical College, Dhaka & Obst and Gynae \\
\hline 300391 & Mst. Israt Jahan & Rangpur Medical College, Rangpur & Obst and Gynae \\
\hline 300394 & Farhana Khanam & Faridpur Medical College, Faridpur & Obst and Gynae \\
\hline 300409 & Nusrat Jahan & Dinajpur Medical College, Dinajpur & Obst and Gynae \\
\hline 300416 & Rehena Nasreen & Kumudini Womens; Medical College, Tangail & Obst and Gynae \\
\hline 300418 & Umma Salma & Chittagong Medical College, Chittagong & Obst and Gynae \\
\hline 300422 & Dr. Rukhsana Parvin & Rajshahi Medical College, Rajshahi & Obst and Gynae \\
\hline 300425 & Khodeza Khanam & Rajshahi Medical College, Rajshahi & Obst and Gynae \\
\hline 300429 & Shahnaz Mizan & Chittagong Medical College, Chittagong & Obst and Gynae \\
\hline 300430 & Mumtahena Amir & Dhaka Medical College, Dhaka & Obst and Gynae \\
\hline 300438 & Taslima Akther & Dinajpur Medical College, Dinajpur & Obst and Gynae \\
\hline 300441 & Mohsina Haider & Dhaka Medical College, Dhaka & Obst and Gynae \\
\hline 300444 & Jebunnaher & Mymensingh Medical College, Mymensingh & Obst and Gynae \\
\hline 300449 & Mohammad Khayrul Bashar Khan & Sher-E-Bangla Medical College, Barisal & Obst and Gynae \\
\hline 300467 & Rownok Jahan & Rajshahi Medical College, Rajshahi & Obst and Gynae \\
\hline 300475 & Rifat Sultana & Mymensingh Medical College, Mymensingh & Obst and Gynae \\
\hline 300478 & Subrata Kumar Bagchi & Dhaka Medical College, Dhaka & Obst and Gynae \\
\hline 300479 & Jayanti Rani Dhar & Rajshahi Medical College, Rajshahi & Obst and Gynae \\
\hline 300484 & Kazi Foyeza Akther & Dinajpur Medical College, Dinajpur & Obst and Gynae \\
\hline 300509 & Dr. Ananya Bhattacharjee & Sir Salimullah Medical College, Dhaka & Obst and Gynae \\
\hline 300521 & Sabreena Barkat & Armed Forces Medical College, Dhaka & Obst and Gynae \\
\hline 310002 & Md. Emdad Hussain & Rajshahi Medical College, Rajshahi & Ophthalmology \\
\hline 310004 & Dr. Rajiv Mothey & $\begin{array}{l}\text { University of Science \& Technology } \\
\text { Chittagong (USTC) }\end{array}$ & Ophthalmology \\
\hline 310005 & S M Rejwan Raju & Dhaka Medical College, Dhaka & Ophthalmology \\
\hline 310007 & Most. Sanjida Akter & Rajshahi Medical College, Rajshahi & Ophthalmology \\
\hline 310008 & Tanjila Hossain & Z.H. Sikder Women ¿s Medical College, Dhaka & Ophthalmology \\
\hline 310014 & Mahar Ali & Sher-E-Bangla Medical College, Barisal & Ophthalmology \\
\hline 310017 & Md. Fakhrul Islam & Mymensingh Medical College, Mymensingh & Ophthalmology \\
\hline 310020 & Nazmus Sakeb & Bangladesh Medical College, Dhaka & Ophthalmology \\
\hline 320001 & Md. Talal Mamun & Sappro Dental College, Dhaka & Oral and Maxillofacial Surgery \\
\hline 320002 & Dr. Sumanta Kumar Gain & Dhaka Dental College, Dhaka & Oral and Maxillofacial Surgery \\
\hline 320003 & Mausumi Iqbal & Sappro Dental College, Dhaka & Oral and Maxillofacial Surgery \\
\hline 320004 & Md. Abdul Hakim & Rajshahi Medical College, Rajshahi & Oral and Maxillofacial Surgery \\
\hline
\end{tabular}




\begin{tabular}{|c|c|c|c|}
\hline Roll No. & Name & From where graduated & Subject \\
\hline 320005 & Dr. Ashim Kumar Saha & Dhaka Dental College, Dhaka & Oral and Maxillofacial Surgery \\
\hline 320007 & Sabiha Alam & Bangladesh Dental College, Dhaka & Oral and Maxillofacial Surgery \\
\hline 320008 & $\begin{array}{l}\text { Dr. Shamsul Alam Mohammad } \\
\text { Imran Hossain }\end{array}$ & Sappro Dental College, Dhaka & Oral and Maxillofacial Surgery \\
\hline 320009 & Sidratul Muntaha & Dhaka Dental College, Dhaka & Oral and Maxillofacial Surgery \\
\hline 330001 & Marina Akhtar & Rangpur Dental College, Rangpur & $\begin{array}{l}\text { Orthodontics and Dentofacial } \\
\text { Orthopaedics }\end{array}$ \\
\hline 330017 & Mohammad Lanzur Rahman & Dhaka Dental College, Dhaka & $\begin{array}{l}\text { Orthodontics and Dentofacial } \\
\text { Orthopaedics }\end{array}$ \\
\hline 350005 & Md.Ariful Islam & MAG Osmani Medical College, Sylhet & Otolaryngology \\
\hline 350012 & Dr. Horidas Kumar Paul & Rangpur Medical College, Rangpur & Otolaryngology \\
\hline 350021 & Md.Nesar Uddin & Dhaka Medical College, Dhaka & Otolaryngology \\
\hline 350023 & Md. Aminul Haque & Sher-E-Bangla Medical College, Barisal & Otolaryngology \\
\hline 350027 & Sanjoy Das & Sir Salimullah Medical College, Dhaka & Otolaryngology \\
\hline 390002 & Syeda Humaida Hasan & Chittagong Medical College, Chittagong & Paediatrics \\
\hline 390007 & Farhana Afroze & MAG Osmani Medical College, Sylhet & Paediatrics \\
\hline 390013 & Jahangir Alam & Armed Forces Medical College, Dhaka & Paediatrics \\
\hline 390014 & Saira Khan & Armed Forces Medical College, Dhaka & Paediatrics \\
\hline 390015 & Samia Chharra & Armed Forces Medical College, Dhaka & Paediatrics \\
\hline 390018 & Dr. Rana Kumar Biswas & Rajshahi Medical College, Rajshahi & Paediatrics \\
\hline 390023 & Dr. Tapon Kumar Roy & Rajshahi Medical College, Rajshahi & Paediatrics \\
\hline 390024 & Dilruba Sultana & Khulna Medical College, Khulna & Paediatrics \\
\hline 390035 & Anjana Basak & Shaheed Ziaur Rahman Medical College, Bogra & Paediatrics \\
\hline 390037 & Mahboba Akther & Sir Salimullah Medical College, Dhaka & Paediatrics \\
\hline 390038 & Dr. Shima Bhadra & Chittagong Medical College, Chittagong & Paediatrics \\
\hline 390040 & Dr. Khaleda Begum & Sir Salimullah Medical College, Dhaka & Paediatrics \\
\hline 390042 & $\begin{array}{l}\text { Mohammad Nazmul } \\
\text { Islam Bhuiyan }\end{array}$ & Chittagong Medical College, Chittagong & Paediatrics \\
\hline 390044 & Sanchita Sarker & Rajshahi Medical College, Rajshahi & Paediatrics \\
\hline 390047 & Md Nazmul Hassan & Jahurul Islam Medical College, Bajitpur & Paediatrics \\
\hline 390048 & Salahuddin Ahmed & Dhaka Medical College, Dhaka & Paediatrics \\
\hline 390050 & Dr. Khyrun Nahar & Rangpur Medical College, Rangpur & Paediatrics \\
\hline 390051 & Meftahul Jannat & Dhaka Medical College, Dhaka & Paediatrics \\
\hline 390052 & Dr. Laila Bilqis & Sir Salimullah Medical College, Dhaka & Paediatrics \\
\hline 390055 & Muhammad Ismail Hasan & MAG Osmani Medical College, Sylhet & Paediatrics \\
\hline 390056 & Dr. Md. Mozammel Haque & Rajshahi Medical College, Rajshahi & Paediatrics \\
\hline 390057 & Abu Asma & Comilla Medical College, Comilla & Paediatrics \\
\hline 390058 & Md. Arif Rabbany & Mymensingh Medical College, Mymensingh & Paediatrics \\
\hline 390059 & Mohammad Morshad Alam & MAG Osmani Medical College, Sylhet & Paediatrics \\
\hline 390063 & Shahreen Kabir & Bangladesh Medical College, Dhaka & Paediatrics \\
\hline 390064 & Tasnim Ahmed & Sir Salimullah Medical College, Dhaka & Paediatrics \\
\hline 390069 & Md. Iqbal Hossain & Rajshahi Medical College, Rajshahi & Paediatrics \\
\hline 390070 & Tithi Islam & Mymensingh Medical College, Mymensingh & Paediatrics \\
\hline 390071 & Nahid Farzana & Chittagong Medical College, Chittagong & Paediatrics \\
\hline
\end{tabular}




\begin{tabular}{|c|c|c|c|}
\hline Roll No. & Name & From where graduated & Subject \\
\hline 390072 & Most. Sabina Yasmin & Dhaka Medical College, Dhaka & Paediatrics \\
\hline 390075 & Rubina Farzana & Mymensingh Medical College, Mymensingh & Paediatrics \\
\hline 390076 & Farhana Afroz & Dhaka National Medical College, Dhaka & Paediatrics \\
\hline 390090 & Sabita Rani Das & Chittagong Medical College, Chittagong & Paediatrics \\
\hline 390091 & Rumi Myedull Hossain & Dhaka Medical College, Dhaka & Paediatrics \\
\hline 390099 & Fauzia Nahid & Sher-E-Bangla Medical College, Barisal & Paediatrics \\
\hline 390102 & Mahbuba Tajmila & Rangpur Medical College, Rangpur & Paediatrics \\
\hline 390103 & Rafia Rashid & Sir Salimullah Medical College, Dhaka & Paediatrics \\
\hline 390104 & Sk. Serjina Anwar & Comilla Medical College, Comilla & Paediatrics \\
\hline 390105 & Sadia Khan & Chittagong Medical College, Chittagong & Paediatrics \\
\hline 390108 & Dr. Sabiha Akther & Rajshahi Medical College, Rajshahi & Paediatrics \\
\hline 390114 & Md. Abdul Wadud Sarker & Chittagong Medical College, Chittagong & Paediatrics \\
\hline 390115 & Rubina Afroz Rana & Rangpur Medical College, Rangpur & Paediatrics \\
\hline 390118 & Muhammad Obaidul Haque & Chittagong Medical College, Chittagong & Paediatrics \\
\hline 390119 & Israt Jahan Anny & Rajshahi Medical College, Rajshahi & Paediatrics \\
\hline 390126 & Kuntal Roy & Dinajpur Medical College, Dinajpur & Paediatrics \\
\hline 390127 & Mst. Shanjida Sharmim & Rangpur Medical College, Rangpur & Paediatrics \\
\hline 390128 & Ruhina Tasmeen & Dhaka Medical College, Dhaka & Paediatrics \\
\hline 390129 & Dr. Jobaida Parvin & Sher-E-Bangla Medical College, Barisal & Paediatrics \\
\hline 390132 & Dr. Nasren Akter & Khulna Medical College, Khulna & Paediatrics \\
\hline 390133 & Subir De & $\begin{array}{l}\text { University of Science \& Technology } \\
\text { Chittagong (USTC) }\end{array}$ & Paediatrics \\
\hline 390147 & Farhana Rahman & Jahurul Islam Medical College, Bajitpur & Paediatrics \\
\hline 390159 & Md.Farid Hossain & Chittagong Medical College, Chittagong & Paediatrics \\
\hline 390160 & Dr. Ishrat Jahan & Medical College for Women and Hospital, Dhak & ka Paediatrics \\
\hline 390161 & Dr. Tahmina Islam & Dhaka Medical College, Dhaka & Paediatrics \\
\hline 390168 & S.M. Nurun Nabi & Mymensingh Medical College, Mymensingh & Paediatrics \\
\hline 390171 & Md.Mostafa Zaman & Shaheed Ziaur Rahman Medical College, Bogra & Paediatrics \\
\hline 390176 & Taslima Sultana & Dhaka Medical College, Dhaka & Paediatrics \\
\hline 390177 & Dr. Md. Rustam Ali & Khulna Medical College, Khulna & Paediatrics \\
\hline 390179 & Md . Anwarul Azim & MAG Osmani Medical College, Sylhet & Paediatrics \\
\hline 390192 & Biplob Kumer Saha & Mymensingh Medical College, Mymensingh & Paediatrics \\
\hline 390197 & Salma Sultana & Dhaka Medical College, Dhaka & Paediatrics \\
\hline 390198 & Syeda Makluka Morshed & Sher-E-Bangla Medical College, Barisal & Paediatrics \\
\hline 390200 & Md. Homayun Shikdar & Sher-E-Bangla Medical College, Barisal & Paediatrics \\
\hline 390205 & Dr Abu Sayeed Chowdhury & MAG Osmani Medical College, Sylhet & Paediatrics \\
\hline 390206 & Dr Shamima Yeasmin & Rajshahi Medical College, Rajshahi & Paediatrics \\
\hline 390209 & Masuma Akter & Sher-E-Bangla Medical College, Barisal & Paediatrics \\
\hline 390220 & Dazy Barua & Comilla Medical College, Comilla & Paediatrics \\
\hline 400006 & Fahad Islam & BGC Trust Medical College, Chittagong. & Physical Medicine \& Rehabilitation \\
\hline 400007 & Selina Nazir & Chittagong Medical College, Chittagong & Physical Medicine \& Rehabilitation \\
\hline 400008 & Musa Muhammad Hojaifa & Sher-E-Bangla Medical College, Barisal & Physical Medicine \& Rehabilitation \\
\hline 400009 & $\begin{array}{l}\text { Lt. Col. (Dr.) S.M. Shahidul } \\
\text { Haque }\end{array}$ & Sher-E-Bangla Medical College, Barisal & Physical Medicine \& Rehabilitation \\
\hline
\end{tabular}




\begin{tabular}{|c|c|c|c|}
\hline Roll No. & Name & From where graduated & Subject \\
\hline 400010 & Aparajeya Bivab Bikash Baral & Chittagong Medical College, Chittagong & Physical Medicine \& Rehabilitation \\
\hline 420001 & Kamrun Nahar Naly & Bangladesh Dental College, Dhaka & Prosthodontics \\
\hline 430002 & Dr. Md. Harunur Rashid & Bangladesh Medical College, Dhaka & Psychiatry \\
\hline 430004 & Bushra Sultana & Dhaka Medical College, Dhaka & Psychiatry \\
\hline 450005 & Rizwana Rahim Chowdhury & Sindh Medical College, Karachi, Pakistan & Radiology \& Imaging \\
\hline 450009 & Sumi Datta & Chittagong Medical College, Chittagong & Radiology \& Imaging \\
\hline 450010 & Mohammad Anwar Hossain & Rajshahi Medical College, Rajshahi & Radiology \& Imaging \\
\hline 450011 & Farah Nazlee & MAG Osmani Medical College, Sylhet & Radiology \& Imaging \\
\hline 450012 & Sohely Sultana & Mymensingh Medical College, Mymensingh & Radiology \& Imaging \\
\hline 460001 & Md. Ishtiaque Alam & Jalalabad Ragib-Rabeya Medical College, Sylhet & Radiotherapy \\
\hline 460003 & Shamima Afroz Trina & Comilla Medical College, Comilla & Radiotherapy \\
\hline 460004 & Ishrat Sultana & Rajshahi Medical College, Rajshahi & Radiotherapy \\
\hline 460005 & Shaila Sharmin & Rangpur Medical College, Rangpur & Radiotherapy \\
\hline 460007 & Mohammed Mehbub Ahsan Rony & MAG Osmani Medical College, Sylhet & Radiotherapy \\
\hline 480002 & Md. Abu Imran & Chittagong Medical College, Chittagong & Surgery \\
\hline 480010 & Saiful Islam Khan & Khulna Medical College, Khulna & Surgery \\
\hline 480021 & Sardar Shahnabi Jafran & Bangladesh Medical College, Dhaka & Surgery \\
\hline 480029 & Dr. Mohammod Ali & Sher-E-Bangla Medical College, Barisal & Surgery \\
\hline 480034 & Md. Mahfuzul Momen & MAG Osmani Medical College, Sylhet & Surgery \\
\hline 480035 & Md Ariful Islam & Sir Salimullah Medical College, Dhaka & Surgery \\
\hline 480038 & Rubaiya Reza Tumpa & Bangladesh Medical College, Dhaka & Surgery \\
\hline 480043 & Md. Masudar Rahman & Rangpur Medical College, Rangpur & Surgery \\
\hline 480046 & Subrata Sarker & Mymensingh Medical College, Mymensingh & Surgery \\
\hline 480048 & $\begin{array}{l}\text { Abu Kawsar Mohammed } \\
\text { Naser Mazumder }\end{array}$ & Chittagong Medical College, Chittagong & Surgery \\
\hline 480051 & Md. Harun-Or-Rashid & Mymensingh Medical College, Mymensingh & Surgery \\
\hline 480052 & Dr. Mohd. Sultanul Abedin & Rajshahi Medical College, Rajshahi & Surgery \\
\hline 480057 & Mohammad Shahriar Faisal & Mymensingh Medical College, Mymensingh & Surgery \\
\hline 480063 & Mohammad Al-Mamun & Comilla Medical College, Comilla & Surgery \\
\hline 480064 & Md. Meshkat Uddin Khan & Dinajpur Medical College, Dinajpur & Surgery \\
\hline 480068 & Habibun Nobi Md Shafiquzzaman & Khulna Medical College, Khulna & Surgery \\
\hline 480069 & Apurba Kishore Paul & MAG Osmani Medical College, Sylhet & Surgery \\
\hline 480074 & Rafiul Karim Khan & Mymensingh Medical College, Mymensingh & Surgery \\
\hline 480082 & U Than Kyow & Dinajpur Medical College, Dinajpur & Surgery \\
\hline 480086 & Shamol Kumar Paul & Khulna Medical College, Khulna & Surgery \\
\hline 480087 & Liton Kumer Shaha & Sir Salimullah Medical College, Dhaka & Surgery \\
\hline 480089 & Dr. Md. Ekramul Haque & MAG Osmani Medical College, Sylhet & Surgery \\
\hline 480090 & Surjit Ghosh & MAG Osmani Medical College, Sylhet & Surgery \\
\hline 480092 & Mohammad Saif Uddin & MAG Osmani Medical College, Sylhet & Surgery \\
\hline 480094 & Abdullah Al Mamun Chowdhury & Rangpur Medical College, Rangpur & Surgery \\
\hline 480095 & Dr Mohammad Monir Hossain & Rajshahi Medical College, Rajshahi & Surgery \\
\hline 480106 & Nur Mohammod Sayed Bin Aziz & Mymensingh Medical College, Mymensingh & Surgery \\
\hline
\end{tabular}




\begin{tabular}{llll}
\hline Roll No. & Na me & From where graduated & Subject \\
\hline 480121 & Mir Rasekh Alam Ovi & Dhaka Medical College, Dhaka & Surgery \\
480122 & Mohammad Kamruzzaman & Dhaka Medical College, Dhaka & Surgery \\
480137 & Eliza Sultana & Rajshahi Medical College, Rajshahi & Surgery \\
480138 & Md Nasir Uddin & MAG Osmani Medical College, Sylhet & Surgery \\
480140 & Sayem Al Monsur Faizi & Mymensingh Medical College, Mymensingh & Surgery \\
480146 & Proshanta Roy & Dinajpur Medical College, Dinajpur & Surgery \\
480147 & Gouranga Kumar Bose & Rajshahi Medical College, Rajshahi & Surgery \\
480151 & Fakir Amirul Islam & Rangpur Medical College, Rangpur & Surgery \\
480175 & Md. Abu Sayem & Sir Salimullah Medical College, Dhaka & Surgery \\
480177 & S. M. Minhajul Hasan Chowdhury & Chittagong Medical College, Chittagong & Surgery \\
480189 & Morshed Ali & Chittagong Medical College, Chittagong & Surgery \\
480190 & Dr. Saurav Sutar & Sher-E-Bangla Medical College, Barisal & Surgery \\
480197 & Sonia Akter & Enam Medical College, Savar, Dhaka & Surgery \\
480200 & Farid Uddin Ahamad & Dhaka Medical College, Dhaka & Surgery \\
480209 & Dr. Faisal Ahme & Chittagong Medical College, Chittagong & Surgery \\
480228 & Dr.Md. Abul Hosen & Rajshahi Medical College, Rajshahi & Surgery \\
480244 & S.M.Syeed-Ul-Alam & Sher-E-Bangla Medical College, Barisal & Surgery \\
480248 & Md. Rabiul Karim & Rangpur Medical College, Rangpur & Surgery \\
480251 & Mohammad Fazlul Haque & MAG Osmani Medical College, Sylhet & Surgery \\
480264 & Fayem Chowdhury & Mymensingh Medical College, Mymensingh & Surgery
\end{tabular}

The following candidates satisfied the Board of Examiners and are declared to have passed the MCPS Examinations held in July, 2016 subject to confirmation by the council of Bangladesh College of Physicians and Surgeons

\begin{tabular}{llll}
\hline Roll No & Name & From Where Graduate & Subject \\
\hline 130011 & Shovan Rahman & Mymensingh Medical College, Mymensingh & Preli - Medicine \\
290001 & Md Amir Ali & Mymensingh Medical College, Mymensingh & Preli - Surgery \\
290002 & Md. Kamal Hossen & Mymensingh Medical College, Mymensingh & Preli - Surgery \\
340004 & Dr. Md. Mizanur Rahman & Shaheed Ziaur Rahman Medical College, Bogra & Preli - Surgery \\
410001 & Mohammad Mominur & Dinajpur Medical College, Dinajpur & Preli - Surgery \\
& Rahman Khan & Rajshahi Medical College, Rajshahi & Preli - Surgery \\
410002 & Sudip Kumar Karmoker & Armed Forces Medical College, Dhaka & Preli - Surgery \\
410005 & Chowdhury Rashedul Mughni & Aro & Preli - Surgery \\
\hline
\end{tabular}

The following candidates satisfied the Board of Examiners and are declared to have passed the MCPS Examinations held in July 2016 subject to confirmation by the council of Bangladesh College of Physicians and Surgeons.

\begin{tabular}{llll}
\hline Roll No & Name & From Where Graduate & Subject \\
\hline 110005 & Md. Mahbubul Alam Sarker & Chittagong Medical College, Chittagong & Anaesthesiology \\
110010 & Nelufa Tahera Rahman & Dhaka Medical College, Dhaka & Anaesthesiology \\
910001 & Masuma Ahmed Salsabil & Dhaka Medical College, Dhaka & Clinical Pathology \\
910003 & Md. Mahbur Rashid Sarker & Shaheed Ziaur Rahman Medical College, Bogra & Clinical Pathology \\
910005 & Samia Mahmood & Armed Forces Medical College, Dhaka & Clinical Pathology \\
910006 & Dr. S. M. Rashidul Kabir & Dhaka Medical College, Dhaka & Clinical Pathology \\
& & & \\
\hline
\end{tabular}




\begin{tabular}{|c|c|c|c|}
\hline Roll No. & Name & From where graduated & Subject \\
\hline 910007 & Fatema Akter & Armed Forces Medical College, Dhaka & Clinical Pathology \\
\hline 910009 & Dr. Fahad Mohammad Sadat & MAG Osmani Medical College, Sylhet & Clinical Pathology \\
\hline 930001 & Md. Jewel Rana & Dhaka Dental College, Dhaka & Dental Surgery \\
\hline 930005 & Mohammad Rezaul Karim Ripon & Dhaka Dental College, Dhaka & Dental Surgery \\
\hline 930008 & Md. Ataul Gani Sarker & Dhaka Dental College, Dhaka & Dental Surgery \\
\hline 160004 & Md. Abdullah-Al Mamun & Sir Salimullah Medical College, Dhaka & Dermatology and Venereology \\
\hline 160010 & Ayesha Siddika & Sher-E-Bangla Medical College, Barisal & Dermatology and Venereology \\
\hline 240038 & Adnan Bashar & Mymensingh Medical College, Mymensingh & Medicine \\
\hline 240044 & S.M.Showkat Ali & \multicolumn{2}{|c|}{ University of Science \& Technology Chittagong (USTC) Medicine } \\
\hline 240074 & Md. Mainuddin Sohel & Chittagong Medical College, Chittagong & Medicine \\
\hline 240083 & Mohmmed Tauseef Sharip & Chittagong Medical College, Chittagong & Medicine \\
\hline 240116 & Dr. A.B.M. Shafiuzzaman & Mymensingh Medical College, Mymensingh & Medicine \\
\hline 240118 & Ahsan Ullah & Chittagong Medical College, Chittagong & Medicine \\
\hline 240122 & Md Anamul Haque & Sir Salimullah Medical College, Dhaka & Medicine \\
\hline 240130 & Taslima Akter & Chittagong Medical College, Chittagong & Medicine \\
\hline 240136 & Prodip Kumar Sarkar & Dhaka Medical College, Dhaka & Medicine \\
\hline 240137 & Dr Md Saiful Islam Patwary & Sher-E-Bangla Medical College, Barisal & Medicine \\
\hline 300004 & Nishat Anam Borna & Rajshahi Medical College, Rajshahi & Obst and Gynae \\
\hline 300016 & Dr. Maya Rani Das & Sir Salimullah Medical College, Dhaka & Obst and Gynae \\
\hline 300019 & Dr. Sharmin Sultana & Rajshahi Medical College, Rajshahi & Obst and Gynae \\
\hline 300020 & Md. Janibul Haque & Dhaka Medical College, Dhaka & Obst and Gynae \\
\hline 300028 & Dr. Munthasir Yeashmin & MAG Osmani Medical College, Sylhet & Obst and Gynae \\
\hline 300038 & Basabi Roy & Khulna Medical College, Khulna & Obst and Gynae \\
\hline 300056 & Khadiza Siddique & Mymensingh Medical College, Mymensingh & Obst and Gynae \\
\hline 300060 & Meem Shahrin & Dhaka Medical College, Dhaka & Obst and Gynae \\
\hline 300067 & Hafiza Farzana & Sher-E-Bangla Medical College, Barisal & Obst and Gynae \\
\hline 300090 & Dr. Mst. Anjuman Ara & Kumudini Womens; Medical College, Tangail & Obst and Gynae \\
\hline 300092 & Dr. Fahmida Yesmin & Sir Salimullah Medical College, Dhaka & Obst and Gynae \\
\hline 310009 & Subrina Afrin & Ibrahim Medical College, Dhaka & Ophthalmology \\
\hline 310017 & Dr. S.M. Mosaddeka Islam & Armed Forces Medical College, Dhaka & Ophthalmology \\
\hline 310018 & Sumaiya Sultana Binte Mosharraf & Armed Forces Medical College, Dhaka & Ophthalmology \\
\hline 310019 & Tahmina Sultana & Armed Forces Medical College, Dhaka & Ophthalmology \\
\hline 310021 & Hasan Jawad & Khulna Medical College, Khulna & Ophthalmology \\
\hline 310022 & Mst. Mahbubay Habibah & Rajshahi Medical College, Rajshahi & Ophthalmology \\
\hline 310023 & Tazbir Ahmed & Ibrahim Medical College, Dhaka & Ophthalmology \\
\hline 310025 & Md. Abdur Rashid & Khulna Medical College, Khulna & Ophthalmology \\
\hline 350005 & Ummey Ayeman Sharmin & Armed Forces Medical College, Dhaka & Otolaryngology \\
\hline 430002 & Fahmida Ferdous & Dhaka National Medical College, Dhaka & Psychiatry \\
\hline 450002 & Mosammat Arina Sultana & Rajshahi Medical College, Rajshahi & Radiology \& Imaging \\
\hline 450004 & Md. Faijus Saleheen & Shaheed Mansur Ali Medical College, Dhaka & Radiology \& Imaging \\
\hline 450006 & Md. Tarif Al Mostakim & Armed Forces Medical College, Dhaka & Radiology \& Imaging \\
\hline 460002 & Samina Islam & Comilla Medical College, Comilla & Radiotherapy \\
\hline 480023 & Md. Saiful Islam Khan & Rangpur Medical College, Rangpur & Surgery \\
\hline 480043 & Mohammad Aminul Islam & Chittagong Medical College, Chittagong & Surgery \\
\hline 480044 & Quazi Sabran Uddin Ahmed & Rajshahi Medical College, Rajshahi & Surgery \\
\hline
\end{tabular}

